The Astrophysical Journal, 487:L143-L146, 1997 October 1

(C) 1997. The American Astronomical Society. All rights reserved. Printed in U.S.A.

\title{
MULTIWAVELENGTH OBSERVATIONS OF A FLARE FROM MARKARIAN 501
}

\author{
M. Catanese, ${ }^{1}$ S. M. Bradbury, ${ }^{2}$ A. C. Breslin, ${ }^{3}$ J. H. Buckley, ${ }^{4}$ D. A. Carter-Lewis, ${ }^{1}$ M. F. Cawley, \\ C. D. Dermer, ${ }^{6}$ D. J. Fegan, ${ }^{3}$ J. P. Finley, ${ }^{7}$ J. A. Gaidos, ${ }^{7}$ A. M. Hillas, ${ }^{2}$ W. N. Johnson, ${ }^{6}$ \\ F. Krennrich, ${ }^{1}$ R. C. Lamb ${ }^{8}$ R. W. Lessard, ${ }^{7}$ D. J. Macomb,${ }^{9,10}$ J. E. McEnery, ${ }^{3}$ \\ P. Moriarty, ${ }^{11}$ J. Quinn, ${ }^{3,4}$ A. J. Rodgers, ${ }^{2}$ H. J. Rose, ${ }^{2}$ F. W. Samuelson, ${ }^{1}$ \\ G. H. Sembroski, ${ }^{7}$ R. Srinivasan, ${ }^{7}$ T. C. Weekes, ${ }^{4}$ and J. Zweerink ${ }^{1}$ \\ Received 1997 June 13; accepted 1997 July 15; published 1997 September 2
}

\begin{abstract}
We present multiwavelength observations of the BL Lacertae object Markarian 501 (Mrk 501) in 1997 between April 8 and April 19. Evidence of correlated variability is seen in very high energy (VHE; $E \gtrsim 350 \mathrm{GeV}$ ) $\gamma$ ray observations taken with the Whipple Observatory $\gamma$-ray telescope, data from the Oriented Scintillation Spectrometer Experiment of the Compton Gamma Ray Observatory, and quick-look results from the All-Sky Monitor of the Rossi X-Ray Timing Explorer, while EGRET did not detect Mrk 501. Short-term optical correlations are not conclusive, but the $U$-band flux observed with the $1.2 \mathrm{~m}$ telescope of the Whipple Observatory was $10 \%$ higher than in March. The average energy output of Mrk 501 appears to peak in the 2-100 keV range, which suggests an extension of the synchrotron emission to at least $100 \mathrm{keV}$, the highest observed in a blazar and $\sim 100$ times higher than that seen in the other TeV-emitting BL Lac object, Mrk 421. The VHE $\gamma$-ray flux observed during this period is the highest ever detected from this object. The VHE $\gamma$-ray energy output is somewhat lower than the 2-100 keV range, but the variability amplitude is larger. The correlations seen here do not require relativistic beaming of the emission unless the VHE spectrum extends to $\gtrsim 5 \mathrm{TeV}$.
\end{abstract}

Subject headings: BL Lacertae objects: individual (Markarian 501) — gamma rays: observations

\section{INTRODUCTION}

Markarian 501 (Mrk 501) is a BL Lacertae (BL Lac) object at $z=0.033$ (see, e.g., Stickel, Fried, \& Kühr 1993), which makes it the second closest known BL Lac after Mrk 421. Mrk 501 was discovered as a $\gamma$-ray source at $E>300 \mathrm{GeV}$ by the Whipple collaboration (Quinn et al. 1996) and recently confirmed by the HEGRA collaboration (Bradbury et al. 1997). Its initial detection level was $8 \%$ of the very high energy (VHE) flux from the Crab Nebula. Like the other TeV-emitting active galactic nuclei, Mrk 421, Mrk 501 exhibits day-scale variability in its VHE $\gamma$-ray emission (Quinn et al. 1996), but prior to 1997, the $\gamma$-ray emission from Mrk 421 was generally observed to have a higher mean flux (Schubnell et al. 1996), be more frequently variable (Buckley et al. 1996), and exhibit higher amplitude and shorter timescale flares (Gaidos et al. 1996). EGRET has never detected Mrk 501 at a statistically significant level (Fichtel et al. 1994; Thompson et al. 1995). Its low VHE flux and the nearby EGRET source, 4C 38.41 (Mattox et al. 1993), were previously assumed to account for this.

\footnotetext{
${ }^{1}$ Department of Physics and Astronomy, Iowa State University, Ames, IA 50011-3160

${ }^{2}$ Department of Physics, University of Leeds, Leeds LS2 9JT, Yorkshire, England, UK.

${ }^{3}$ Experimental Physics Department, University College, Belfield, Dublin 4, Ireland.

${ }^{4}$ Fred Lawrence Whipple Observatory, Harvard-Smithsonian Center for Astrophysics, P.O. Box 97, Amado, AZ 85645-0097.

${ }^{5}$ Physics Department, St. Patrick's College, Maynooth, County Kildare, Ireland.

${ }^{6}$ E. O. Hulburt Center for Space Research, Code 7650, Naval Research Laboratory, Washington, DC 20375.

${ }^{7}$ Department of Physics, Purdue University, West Lafayette, IN 47907.

${ }^{8}$ Space Radiation Laboratory, California Institute of Technology, Pasadena, CA 91125.

${ }^{9}$ NASA/Goddard Space Flight Center, Code 662, Greenbelt, MD 20771.

${ }^{10}$ Universities Space Research Association.

${ }^{11}$ Department of Physical Sciences, Regional Technical College, Galway, Ireland.
}

Here we present Whipple Observatory VHE $\gamma$-ray and optical, Oriented Scintillation Spectrometer Experiment (OSSE), and EGRET observations of Mrk 501 during a flare in 1997 April. We also include quick-look X-ray results from the AllSky Monitor (ASM). Overlapping observations of Mrk 501 with the Rossi X-Ray Timing Explorer (RXTE) (Catanese 1997) and BeppoSAX (Pian et al. 1997) are reported elsewhere. Details of the observations are given in $\S 2$. They show evidence of a correlation among the wave bands covered by the above experiments (see $\S 3$ ), except EGRET, which detected no emission from Mrk 501. The implications of these results are discussed in $\S 4$.

\section{OBSERVATIONS AND ANALYSIS}

\subsection{Whipple Observatory Observations}

The VHE observations reported in this Letter were made with the atmospheric Cerenkov imaging technique (Cawley \& Weekes 1995) using the $10 \mathrm{~m}$ optical reflector located at the Whipple Observatory on Mount Hopkins in Arizona (elevation $2.3 \mathrm{~km}$ ) (Cawley et al. 1990). A 151 photomultiplier tube (upgraded in 1996 from 109) camera mounted in the focal plane of the reflector records images of atmospheric Cerenkov radiation from air showers produced by $\gamma$-rays and cosmic rays. The $\gamma$-ray selection was based on the Supercuts criteria (Reynolds et al. 1993); however, the camera upgrade necessitated that the analysis cuts be changed slightly (Weekes et al. 1997). The data reported in this Letter are analyzed with a tracking analysis (see Quinn et al. 1996).

Count rates for $\gamma$-rays are converted to integral fluxes by expressing them as a multiple of the Crab Nebula count rate and then multiplying that fraction by the Crab Nebula flux: $I$ $(>350 \mathrm{GeV})=1.05 \pm 0.24 \times 10^{-10}$ photons cm $\mathrm{cm}^{-2} \mathrm{~s}^{-1}$ (Hillas et al. 1997). The errors in the absolute fluxes listed below are in general dominated by the uncertainty in the Crab Nebula flux. The statistical significance of the detections are more ac- 


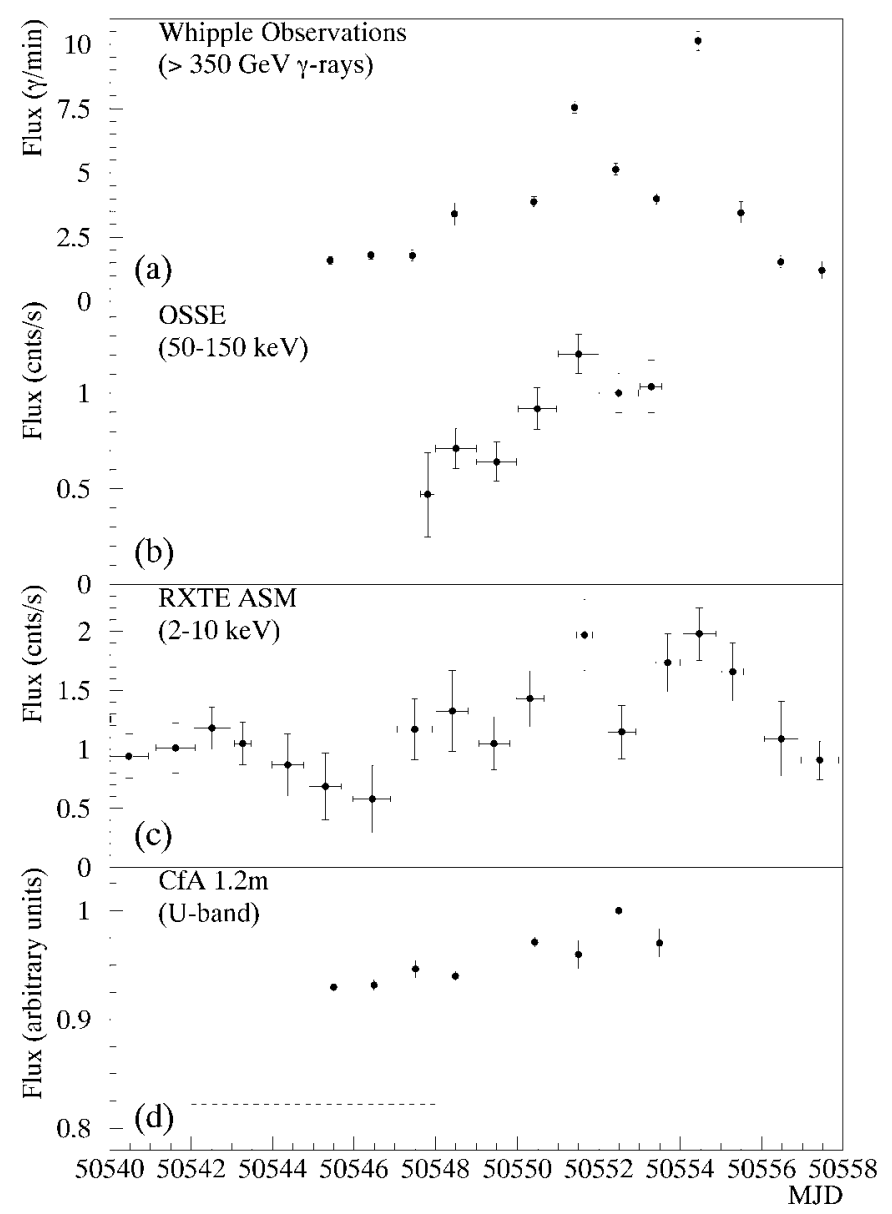

FIG. 1.-(a) VHE $\gamma$-ray, (b) OSSE 50-150 keV, (c) ASM 2-10 keV, and (d) $U$-band optical light curves of Mrk 501 for the period 1997 April 2 (MJD 50540) to 1997 April 20 (MJD 50558). The dashed line in (d) indicates the average $U$-band flux in 1997 March.

curately indicated by the $\gamma$-ray count rates (see Fig. $1 a$ ). This procedure assumes that the VHE $\gamma$-ray flux of the Crab Nebula is steady, as $7 \mathrm{yr}$ of Whipple Observatory data indicate (Hillas et al. 1997), and that the Mrk 501 spectrum is identical to that of the Crab Nebula between 0.3 and $10 \mathrm{TeV}, d N / d E \propto E^{-2.5}$ (Hillas et al. 1997), which may not be the case. Estimates of the VHE spectrum of Mrk 501 will be undertaken in future work.

VHE observations of Mrk 501 were taken nightly April 7-19, for a total exposure of $19.9 \mathrm{hr}$. The mean flux above 350 $\mathrm{GeV}$ during this period was $16.4 \pm 3.9 \times 10^{-11}$ photons $\mathrm{cm}^{-2}$ $\mathrm{s}^{-1}$, which is $\sim 1.6$ times the flux of the Crab Nebula. By comparison, the $\gamma$-ray flux from Mrk 501 was never recorded to be higher than 0.5 times the Crab Nebula flux in previous seasons (Quinn et al. 1996). The flux during April ranged from a low of $4.9 \pm 1.8 \times 10^{-11}$ photons $\mathrm{cm}^{-2} \mathrm{~s}^{-1}$ on April 19 to a high of $40.5 \pm 9.6 \times 10^{-11}$ photons $\mathrm{cm}^{-2} \mathrm{~s}^{-1}$ on April 16 The latter is the highest flux recorded from Mrk 501 by the Whipple Observatory. No evidence of hour-scale variability was found in this data set.

Optical observations were taken April 7-15 with the $1.2 \mathrm{~m}$ Ritchey-Chrétien telescope of the Whipple Observatory with standard UBVRI filters. The $12^{\prime}$ full-field CCD frames were analyzed by means of relative photometry with a $6 "$ aperture applied to Mrk 501 and several comparison stars in the field of view. The $U$-band fluxes (see Fig. 1d) are expressed in
TABLE 1

OSSE Flux Measurements FOr MrK 501

\begin{tabular}{|c|c|c|c|}
\hline & $0.05-0.15 \mathrm{MeV}$ & $0.15-0.50 \mathrm{MeV}$ & $0.5-3.5 \mathrm{MeV}$ \\
\hline Flux ...... & $24.2 \pm 1.3$ & $2.0 \pm 0.3$ & $<0.2$ \\
\hline
\end{tabular}

arbitrary units with no galaxy light subtraction. These results are described in detail by Buckley \& McEnery (1997).

\subsection{Compton Gamma Ray Observatory Observations}

The Compton Gamma Ray Observatory (CGRO) observed Mrk 501 for the period April 9-15 as the result of a Target of Opportunity initiated in response to the reported high level of VHE $\gamma$-ray activity (Breslin et al. 1997). We report here on the measurements of the OSSE and EGRET instruments on CGRO.

OSSE observes $\gamma$-rays in the $0.05-10 \mathrm{MeV}$ energy range. A description of OSSE and its performance and analysis procedures is given by Johnson et al. (1993). For the observation of Mrk 501, the CGRO viewing attitude and the OSSE observing sequence were selected to avoid the X-ray binary Hercules X-1 and the EGRET source 4C 38.41. This was achieved by viewing Mrk $501 \sim 0.6$ off-axis, or at $\sim 80 \%$ of maximum response, and by making background observations that were offset by \pm 7.5 .

The OSSE data taken during this 6 day period yielded a very strong detection of Mrk 501 (see Table 1). The daily flux observed by OSSE varied by over a factor of 2, peaking on April 13 (see Fig. 1). Mrk 501 has not previously been observed by OSSE, so no comparison to previous flux levels can be made. The average 50-150 keV flux detected from Mrk 501 is higher than the emission seen by OSSE from any other blazar (McNaron-Brown et al. 1995), except the highest recorded state from 3C 273 (McNaron-Brown et al. 1996). We note that it may not be strictly valid to compare Mrk 501 to 3C 273 because the latter object is not a typical blazar. The average of the OSSE data for this 6 day observation is well fitted by a single power law in the energy range $0.05-2.0 \mathrm{MeV}\left(\chi^{2}=0.794,64\right.$ degrees of freedom) of the form

$$
N(E)=1.8 \pm 0.1 \times 10^{-2}\left(\frac{E}{0.1 \mathrm{MeV}}\right)^{-2.08 \pm 0.15}
$$

photons $\mathrm{cm}^{-2} \mathrm{~s}^{-1} \mathrm{MeV}^{-1}$, where the uncertainties represent the $68 \%$ confidence interval for joint variation of two parameters $\left(\Delta \chi^{2}=2.3\right)$. The spectral index is somewhat steep compared with OSSE spectra of other blazars, but it is still within the 1.0-2.1 range reported by McNaron-Brown et al. (1995). There was no statistically significant variation in the power-law index with time when fitting power-law spectra to the six daily average OSSE measurements.

The EGRET instrument (see, e.g., Kanbach et al. 1988; Thompson et al. 1993) is sensitive to $\gamma$-rays in the energy range spanning approximately $30 \mathrm{MeV}-30 \mathrm{GeV}$. The EGRET data were analyzed with maximum likelihood techniques (Mattox et al. 1996), taking into account the contributions of isotropic and Galactic diffuse emission (Hunter et al. 1997) and other bright sources near the position of Mrk 501. The observations taken April 9-15 indicate an excess of 1.5 $\sigma$, which corresponds to a $2 \sigma$ upper limit of $I(>100 \mathrm{MeV})<3.6 \times 10^{-7}$ photons $\mathrm{cm}^{-2} \mathrm{~s}^{-1}$. 


\section{RESULTS}

Figure 1 shows daily flux levels for the contemporaneous observations of Mrk 501. The average flux level in the $U$-band in March is also included in the figure (Fig. 1d, dashed line) to indicate the significant $(\gtrsim 10 \%)$ increase in flux between March and April. An 11 day rise and fall in flux is evident in the VHE and ASM wave bands, with peaks on April 13 and 16. The 50-150 keV flux detected by OSSE also increases between April 9 and 15, with a peak on April 13. The optical data may show a correlated rise, but the variation is small (at most 6\%). Subtraction of the galaxy light contribution will increase the amplitude of this variation, but it should still remain lower than in $\mathrm{X}$-rays, given that the $R$-band contribution of the galaxy light is $\sim 75 \%$ (Wurtz, Stocke, \& Yee 1996), and the $U$-band contribution should be much less. The ratio of the fluxes between April 13 and April 9 are 4.2, 2.6, 1.7, and 1.01 for the VHE, OSSE, ASM, and $U$-band emissions, respectively.

In Figure 2 we show the spectral energy distribution, plotted as the power per logarithmic bandwidth, $\nu F_{\nu}$, versus frequency, $\nu$. The average flux for April 9-15, the duration of the CGRO Target of Opportunity, is indicated by the filled circles. The filled square indicates the peak VHE $\gamma$-ray flux from April 16. The OSSE data for this plot have been divided into eight energy bands between $50 \mathrm{keV}$ and $470 \mathrm{keV}$. No excess emission is seen above $470 \mathrm{keV}$, so those data are not shown. The VHE integral fluxes are converted to single point fluxes by assuming that the spectrum follows a power law with the same spectral index as the Crab Nebula. The EGRET integral flux upper limit is converted to a single point flux limit assuming that $d N / d E$ $\propto E^{-2}$. The ASM 2-10 keV count rate is converted to a flux point by normalizing to the Crab flux and assuming that Mrk 501 has the same photon spectrum as the Crab $\left(E^{-2.05}\right)$ within that energy range. From Figure 2, a peak in the power output occurs in the $2-100 \mathrm{keV}$ range, and the VHE $\gamma$-ray power output is much higher than at EGRET energies but somewhat below that in the $2-100 \mathrm{keV}$ range.

\section{DISCUSSION}

The results presented here show that for Mrk 501, like Mrk 421 (Buckley et al. 1996; Macomb et al. 1995), the VHE $\gamma$ rays and the soft $\mathrm{X}$-rays vary together, the energy budget at $\mathrm{X}$-rays and $\gamma$-rays is comparable, there is evidence of correlated optical variability, and the cutoff in the synchrotron emission occurs at much higher energies than in radio-selected BL Lac objects or flat-spectrum radio quasars. However, Mrk 421 has a large deficit in energy output between $1 \mathrm{keV}$ and $50 \mathrm{keV}$ (McNaron-Brown et al. 1995; Buckley et al. 1996). With Mrk 501 , there is instead a peak in the power output at $\sim 100 \mathrm{keV}$, and the deficit has shifted to between $1 \mathrm{MeV}$ and $350 \mathrm{GeV}$. Also, the variability amplitude for the VHE $\gamma$-rays is larger than the X-ray and OSSE variations, whereas similar flares in Mrk 421 showed comparable amplitude variations (Buckley et al. 1996; Macomb et al. 1995).

A likely explanation of the OSSE detection is that the synchrotron emission in Mrk 501 extends to $100 \mathrm{keV}$, compared with the $\sim 1 \mathrm{keV}$ cutoff seen in Mrk 421. If this is synchrotron emission from nonthermal electrons, then the maximum electron Lorentz factor is given by $\gamma_{\max } \cong\left(3 \times 10^{6}\right)\left(E_{\mathrm{syn}} / B \delta\right)^{1 / 2}$, where $B$ is the magnetic field strength in gauss, $\delta$ is the Doppler factor of the radiating plasma, and $E_{\text {syn }}$ is the cutoff energy of the synchrotron spectrum in units of $100 \mathrm{keV}$. The correlated variability between the hard X-rays and the VHE $\gamma$-rays implies that the same nonthermal electrons are responsible for the two

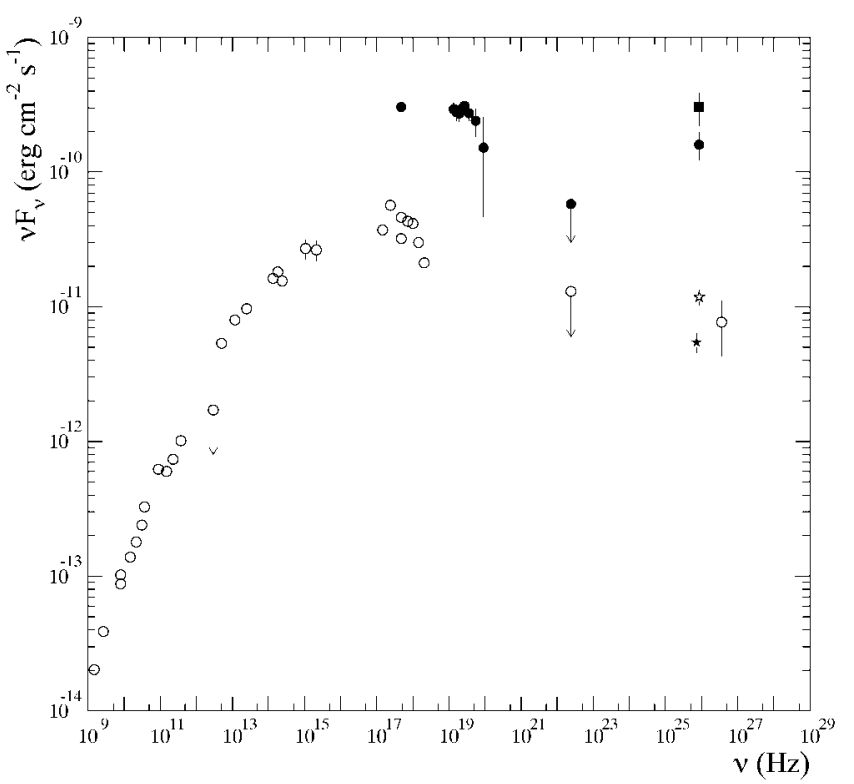

FIG. 2.-The spectral energy distribution of Mrk 501. The contemporaneous observations taken April 9-15 (filled circles), archival data (open circles) (Gear et al. 1994; Owen, Spangler, \& Cotton 1980; Impey \& Neugebauer 1988; Kotilainen et al. 1992; Edelson et al. 1992; Singh \& Garmire 1985; Staubert et al. 1986; Fichtel et al. 1994; Bradbury et al. 1997), the mean VHE $\gamma$-ray flux in 1995 (filled star) (Quinn et al. 1996), the mean flux in 1996 (open star), and the maximum VHE $\gamma$-ray flux detected on April 16 (square) are indicated.

emission components (cf. Macomb et al. 1995), with the VHE $\gamma$-rays produced by Compton scattering of either internal synchrotron photons (e.g., Königl 1981; Maraschi, Ghisellini, \& Celotti 1992; Bloom \& Marscher 1996) or photons produced external to the jet (e.g., Dermer, Schickeiser, \& Mastichiadis 1992; Blandford 1993; Sikora, Begelman, \& Rees 1994). For low-redshift objects, electrons must have Lorentz factors of $\gamma>\left(2 \times 10^{6}\right)\left(E_{\mathrm{C}} / \delta\right)$ in order to Compton scatter photons to energies of $E_{\mathrm{C}}(\mathrm{TeV})$. Thus, the magnetic field $B \lesssim$ $2 E_{\mathrm{syn}}(100 \mathrm{keV}) \delta /\left[E_{\mathrm{C}}(\mathrm{TeV})\right]^{2}$ (see also Buckley et al. 1997).

A lower limit to the magnetic field is implied by the variability of the synchrotron emission. Electrons cool through synchrotron losses in the comoving frame on a timescale of $\Delta t_{\text {syn }}=\left(7.7 \times 10^{8}\right) /\left(B^{2} \gamma\right)$ s. The source might intrinsically vary on a longer timescale, so a lower limit to the observed variability timescale for low-redshift objects is given by $\Delta t_{\text {obs }} \gtrsim \Delta t_{\text {syn }} / \delta$. This implies that $B \gtrsim 9000 /\left[\gamma \delta \Delta t_{\text {obs }}(\text { days })\right]^{1 / 2}$. This expression can be written in terms of the observed photon energy $E_{\mathrm{obs}}(\mathrm{eV})$ with the relation $\nu_{\mathrm{obs}}=\left(2.8 \times 10^{6}\right) B \gamma^{2} \delta$ from synchrotron theory. Putting the above constraints together, the allowed magnetic field strength of the emission region is given by

$$
\left(E_{\mathrm{obs}} \delta \Delta t_{\mathrm{obs}}^{2}\right)^{-1 / 3} \lesssim B \lesssim 2 E_{\mathrm{syn}} \delta /\left(E_{\mathrm{C}}\right)^{2}
$$

The ASM $\left(E_{\mathrm{obs}}=2000 \mathrm{eV}\right)$ flux varies on timescales at least as short as 1 day, which implies that $B \gtrsim 0.08 \delta^{-1 / 3}$ G. Such a range of field strengths are expected at the base of a jet from independent considerations (see, e.g., Marscher \& Gear 1985).

Equation (2) provides a new test for beaming in $\gamma$-ray blazars, although it assumes that the high-energy component arises from Compton scattering of photons by the same nonthermal electrons that produce the synchrotron component. If 
the lower limit in equation (2) exceeds the upper limit when $\delta$ is set equal to 1 , then beaming is implied. Here it is especially crucial to determine the cutoff energy at $\mathrm{TeV}$ energies. If the high-energy spectrum of Mrk 501 continues without break to $\gtrsim 5 \mathrm{TeV}$, then beaming is implied.

This beaming test complements $\gamma \gamma$ transparency arguments that also provide lower limits to $\delta$. The $\gamma$-rays with energy greater than $350 \mathrm{GeV}$ interact with the X-ray photons in the extreme relativistic regime of pair production (i.e., $E_{\mathrm{VHE}} E_{\mathrm{X}} \gg$ $m_{e}^{2} c^{4}$ ), which has been treated by Dermer \& Gehrels (1995). One finds that $\tau_{\gamma \gamma} \lesssim 0.1$ for this interaction, so no beaming is required. Improved limits to the Doppler factor through $\gamma \gamma$ transparency arguments and from equation (2) will be obtained if short timescale correlations are detected between VHE $\gamma$ rays and photons in the Extreme Ultraviolet Explorer or optical regime. Also, if the spectrum observed with OSSE extends beyond $0.511 \mathrm{MeV}, \gamma \gamma$ transparency arguments can be applied to this $\gamma$-ray emission directly (McNaron-Brown et al. 1995).
Finally, it will be important to investigate whether the extension of the synchrotron emission in Mrk 501 to $100 \mathrm{keV}$ is always present or occurred only during the flaring activity shown in this work. Previous compilations of X-ray spectra for Mrk 501 spanning 0.03-30 keV (see, e.g., Ciliegi, Bassani, \& Caroli 1993) are not conclusive, mainly because of the low statistics at high energies. Further studies of Mrk 501 with OSSE and the high-energy instruments on RXTE and BeppoSAX could resolve this issue.

We acknowledge the technical assistance of K. Harris. This research is supported by grants from the U. S. Department of Energy and by NASA, by the Particle Physics and Astronomy Research Council in the UK, and by Forbairt in Ireland. The $\mathrm{X}$-ray data in this work are quick-look results presented by the All-Sky Monitor Rossi X-Ray Timing Explorer team.

\section{REFERENCES}

Blandford, R. D. 1993, in Proc. Compton Symp., ed. M. Friedlander, N. Gehrels, \& D. J. Macomb (New York: AIP), 533

Bloom, S. D., \& Marscher, A. P. 1996, ApJ, 461, 657

Bradbury, S. M., et al. 1997, A\&A, 320, L5

Breslin, A. C., et al. 1997, IAU Circ., 6596

Buckley, J. H., et al. 1996, ApJ, 472, L9 . 1997, Adv. Space. Res., in press

Buckley, J. H., \& McEnery, J. E. 1997, in preparation

Catanese, M. 1997, in preparation

Cawley, M. F., \& Weekes, T. C. 1995, Exp. Astron., 6, 7

Cawley, M. F., et al. 1990, Exp. Astron., 1, 173

Ciliegi, P., Bassani, L., \& Caroli, E. 1993, ApJS, 85, 111

Dermer, C. D., \& Gehrels, N. 1995, ApJ, 447, 103

Dermer, C. D., Schlickeiser, R., \& Mastichiadis, A. 1992, A\&A, 256, L27

Edelson, R., Pike, G. F., Saken, J. M., Kinney, A., \& Shull, J. M. 1992, ApJS, 83,1

Fichtel, C. E., et al. 1994, ApJS, 94, 551

Gaidos, J. A., et al. 1996, Nature, 383, 319

Gear, W. K., et al. 1994, MNRAS, 267, 167

Hillas, A. M., et al. 1997, ApJ, submitted

Hunter, S. D., et al. 1997, ApJ, 481, 205

Impey, C. D., \& Neugebauer, G. 1988, AJ, 95, 307

Johnson, W. N., et al. 1993, ApJS, 86, 693

Kanbach, G., et al. 1988, Space Sci. Rev., 49, 69

Königl, A. 1981, ApJ, 243, 700

Kotilainen, J. K., et al. 1992, MNRAS, 256, 149
Macomb, D. J., et al. 1995, ApJ, 449, L99

Mannheim, K. 1993, A\&A, 269, 67

Maraschi, L., Ghisellini, G., \& Celotti, A. 1992, ApJ, 397, L5

Marscher, A. P., \& Gear, W. K. 1985, ApJ, 298, 114

Mattox, J. R., et al. 1993, ApJ, 410, 609

- 1996, ApJ, 461, 396

McNaron-Brown, K., Johnson, W. N., Dermer, C. D., \& Kurfess, J. D. 1996, ApJ, 474, L85

McNaron-Brown, K., et al. 1995, ApJ, 451, 575

Owen, F. N., Spangler, S. R., \& Cotton, W. D. 1980, AJ, 85, 351

Pian, E., et al. 1997, ApJ, submitted

Quinn, J., et al. 1996, ApJ, 456, L83

Reynolds, P. T., et al. 1993, ApJ, 404, 206

Schönfelder, V., et al. 1996, A\&AS, 120, C13

Schubnell, M. S., et al. 1996, ApJ, 460, 644

Sikora, M., Begelman, M. C., \& Rees, M. J. 1994, ApJ, 421, 153

Singh, K. P., \& Garmire, G. P. 1985, 297, 199

Staubert, R., Bazzano, A., Ubertini, P., Brunner, H., Collmar, W., \& Kendziorra, E. $1986, A \& A, 162,16$

Stickel, M., Fried, J. W., \& Kühr, H. 1993, A\&AS, 98, 393

Takahashi, T., et al. 1996, ApJ, 470, L89

Thompson, D. J., et al. 1993, ApJS, 86, 629 . 1995, ApJS, 101, 259

Weekes, T. C., et al. 1997, in Towards a Major Atmospheric Cherenkov Detector V (Kruger Park), in press

Wurtz, R., Stocke, J. T., \& Yee, H. K. C. 1996, ApJS, 103, 109 